\title{
Mood Disorders Induced by Maternal Overnutrition: The Role of the Gut-Brain Axis on the Development of Depression and Anxiety
}

\begin{abstract}
Jeferson Jantsch ${ }^{1 \dagger}$, Isadora D'Ávila Tassinari ${ }^{2 \dagger}$, Márcia Giovenardi ${ }^{1,3}$, Victorio Bambini-Junior ${ }^{4,5}$, Renata Padilha Guedes ${ }^{1,3}$ and Luciano Stürmer de Fraga ${ }^{2 *}$

${ }^{1}$ Programa de Pós-Graduação em Biociências, Universidade Federal de Ciências da Saúde de Porto Alegre (UFCSPA), Porto Alegre, Brazil, ${ }^{2}$ Programa de Pós-Graduação em Ciências Biológicas: Fisiologia, Universidade Federal do Rio Grande do Sul (UFRGS), Porto Alegre, Brazil, ${ }^{3}$ Programa de Pós-Graduação em Ciências da Saúde, Universidade Federal de Ciências da Saúde de Porto Alegre (UFCSPA), Porto Alegre, Brazil, ${ }^{4}$ School of Pharmacy and Biomedical Sciences, University of Central Lancashire (UCLan), Preston, United Kingdom, ${ }^{5}$ Division of Biomedical and Life Sciences, Faculty of Health and Medicine, Lancaster University, Lancaster, United Kingdom
\end{abstract}

\section{OPEN ACCESS}

Edited by:

Patricia Ybot-Gonzalez, Institute of Biomedicine of Seville (CSIC), Spain

Reviewed by: Evelyn Nunes Goulart da Silva Pereira, Oswaldo Cruz Foundation (Fiocruz),

Brazil

*Correspondence: Luciano Stürmer de Fraga lucianof@ufrgs.br

${ }^{\dagger}$ These authors have contributed equally to this work and share first authorship

Specialty section:

This article was submitted to Cellular Biochemistry,

a section of the journal

Frontiers in Cell and Developmental

Biology

Received: 15 October 2021 Accepted: 10 January 2022 Published: 26 January 2022

Citation: Jantsch J, Tassinari ID, Giovenardi $M$, Bambini-Junior V, Guedes RP and de Fraga LS (2022) Mood Disorders Induced by Maternal Overnutrition: The

Role of the Gut-Brain Axis on the

Development of Depression and Anxiety.

Front. Cell Dev. Biol. 10:795384. doi: 10.3389/fcell.2022.795384
Since the first evidence suggesting that maternal nutrition can impact the development of diseases in the offspring, much has been elucidated about its effects on the offspring's nervous system. Animal studies demonstrated that maternal obesity can predispose the offspring to greater chances of metabolic and neurodevelopmental diseases. However, the mechanisms underlying these responses are not well established. In recent years, the role of the gut-brain axis in the development of anxiety and depression in people with obesity has emerged. Studies investigating changes in the maternal microbiota during pregnancy and also in the offspring demonstrate that conditions such as maternal obesity can modulate the microbiota, leading to long-term outcomes in the offspring. Considering that maternal obesity has also been linked to the development of psychiatric conditions (anxiety and depression), the gut-brain axis is a promising target to be further explored in these neuropsychiatric contexts. In the present study, we review the relationship between maternal obesity and anxious and depressive features, exploring the gut-brain axis as a potential mechanism underlying this relationship.

Keywords: maternal obesity, gut-brain axis, anxiety, depression, microbiota

\section{INTRODUCTION}

The Developmental Origins of Health and Disease (DOHaD) theory brought by Barker and Osmont (1986) states that prenatal environmental factors influence the offspring's health in adulthood (Barker and Osmond, 1986). Both malnutrition and overnutrition are determinants of metabolic and neurological dysfunctions in the offspring, such as impairments in the brain reward circuitry, neuroinflammatory states, and behavioural disorders (Smith and Reyes, 2017).

Neurodevelopmental disorders are well documented in the offspring of obese mothers in humans and animal models (Edlow, 2017; Tong and Kalish, 2021). However, observational epidemiological studies have struggled to establish a direct cause-effect relationship between gestational obesity and neurological diseases in the progeny. Nevertheless, in preclinical studies, there is a substantial body of evidence showing a mechanistic link between maternal obesity and neurological dysfunctions in the offspring (Edlow, 2017). For example, long-term hippocampal insulin resistance in obese mice dams 
was followed by a decrease in the expression of neurogenesis markers and synaptic plasticity in the offspring (Schmitz et al., 2018). Moreover, the impact of maternal obesity is multigenerational, impairing synaptic function in the hippocampus until the third generation (Fusco et al., 2019).

Considering that obesity during pregnancy is increasing globally (Chen et al., 2018), as well as the prevalence of mood disorders such as depression and anxiety (WHO, 2017), it is important to unravel the mechanisms linking these conditions. Maternal obesity alters intestinal microbiota (Chu et al., 2016), which has emerged as a key regulator of metabolism and behaviour. Thus, understanding how the maternal intestinal microbiota influences fetal neurodevelopment is fundamental to comprehend the repercussions of maternal obesity on the mental health of the offspring (Kumpulainen et al., 2018).

This review will discuss the influence of maternal overnutrition on the development of depression and anxiety in the offspring. We will emphasize the role of the gut-brain axis in the mechanisms linking maternal obesity to the harmful neurodevelopment of the progeny.

\section{DEPRESSION}

Intrauterine overnutrition could affect the fetal programming, increasing the risk of the newborn developing neuropsychiatric conditions such as depression (McDonald et al., 2015; Cárdenas-Tueme et al., 2020; Cattane et al., 2021).

Offspring of rodent mothers fed with a high-fat diet (HFD) during pregnancy and lactation shows increased depressivelike behaviour in adolescence and adulthood (Gawlinska et al., 2019), even if the offspring was fed a standard diet. Although there is some controversy in the literature [for review see (Ortiz-Valladares et al., 2021)], it reinforces that prenatal exposure to maternal HFD is a decisive factor that can lead to depressive symptoms later in life (Gawlinska et al., 2019). Transcriptional changes in the prefrontal cortex (Gawlińska et al., 2021), reduction in the number and morphology of dendrites and spines in the hippocampus, amygdala, and somatosensory cortex (Hatanaka et al., 2017), lower volume of brain areas such as the thalamus, hippocampus, nucleus accumbens, and hypothalamus, in addition to changes in synaptophysin and GFAP (glial fibrillary acidic protein) expression (Trujillo-Villarreal et al., 2021) may be part of the mechanisms underlying the behavioural changes seen in the adult offspring.

Maternal exposure to HFD, or a carbohydrate-rich diet, also affects the dopaminergic (DA) system by changing the amount of dopamine and its metabolite DOPAC $(3,4-$ dihydroxyphenylacetic acid) in the nucleus accumbens and the expression of dopamine receptors D1/D2 in the ventral tegmental area (Naef et al., 2008; Naef et al., 2011; Sullivan et al., 2015; Paradis et al., 2017). Dysregulation of these circuits increases the risk of mood disorders and altered function of the mesolimbic DA system, as well as the dopamine receptor in limbic structures, has already been observed in different models of depression (Winter et al., 2007; Tye et al., 2013; Belujon and Grace, 2017).
Clinical studies have reported that elevated levels of proinflammatory cytokines in obese pregnant women can lead to placental dysfunction and are related to neurodevelopmental and neuropsychiatric disorders in the offspring [for review see (Howell and Powell, 2017)]. Evidence from humans and animals also links depressive-like behaviour with immune system disturbances or neuroinflammatory responses. Proinflammatory mediators such as C-reactive protein, interleukin (IL)-6, IL-1 $\beta$, and tumor necrosis factor (TNF)- $\alpha$ affect the development of neural circuits that are critical for regulation of behaviour, providing evidence that inflammation may also be a potential factor leading to increased risk of mood disorders (Rivera et al., 2015; Rasmussen et al., 2019).

Furthermore, emotional changes in the progeny were associated with maternal nutritional status (Ortiz-Valladares et al., 2021) and high maternal body mass index with a decline in psychological development in the early stages of childhood (Bergmann et al., 2016; Aubuchon-Endsley et al., 2017). In summary, poor maternal nutrition during pregnancy negatively impacts neurodevelopmental outcomes in the first years of life and plays an important role in inducing mental disorders in the offspring.

\section{ANXIETY}

Maternal obesity has been associated with offspring's anxiety independently of sex, species, and age (Menting et al., 2019). However, the mechanisms linking maternal obesity to the development of anxiety in the offspring remain a gap in the field. The available evidence will be briefly scrutinized here.

During adolescence, there is an increase in anxiety in the offspring of HFD-fed dams, shown in the light-dark transition task, but a decrease in anxiety in the open field and elevated plusmaze, which could be interpreted as an increased risky behaviour rather than decreased anxiety. Importantly, an increase in the expression of nuclear factor kappa B (NF-kB) and IL-6 genes, responsible for inflammatory responses, was found in the hippocampus of adolescent offspring of dams fed with a hypercaloric diet. But an increase in the expression of antiinflammatory genes such as $\mathrm{IkBa}$ and mitogen-activated protein kinase MKP-1 was also seen, suggesting a disturbance in the expression of inflammatory mediators in the brain of these animals (Sasaki et al., 2014). On the other hand, when analyzing the offspring of HFD-fed dams during adult life, an increase in anxiety-like behaviours was observed in the open field, elevated plus-maze, and light-dark transition tasks. The maternal diet also changed the concentration of plasma corticosteroids after stress challenge and the expression of pro-inflammatory genes in the amygdala, such as NF-kB or IL-6, with a notable sex-specific influence (Sasaki et al., 2013). Although the effect of maternal diet on anxiety did not appear to be influenced by sex or age in the systematic review by Menting et al. (2019), it is clear that this claim is controversial and needs further investigation.

Most studies involving maternal consumption of obesogenic diets use the HFD model. However, the cafeteria diet (CAF) model provides animals with ultra-processed human food 
TABLE 1 | Effects of maternal nutrition on depressive and anxious-like behaviour and reported mechanisms in preclinical studies.

\begin{tabular}{|c|c|c|c|}
\hline Reference & Maternal diet & $\begin{array}{l}\text { Offspring } \\
\text { species and } \\
\text { diet }\end{array}$ & $\begin{array}{l}\text { Molecular and behavioural } \\
\text { findings in the } \\
\text { progeny }\end{array}$ \\
\hline \multicolumn{4}{|c|}{ Depression } \\
\hline Gawlinska et al. (2019) & HFD (60\%) pregnancy and lactation & Wistar rats-SD & $\begin{array}{l}\uparrow \text { Anhedonia and depressive-like behaviour in the FST } \\
\downarrow \text { Irisin in serum and hippocampus of females on PND } 28 \\
\downarrow \text { Interleukin- } 1 \alpha \text { in the hippocampus of females on PND28 and PND63 }\end{array}$ \\
\hline Gawlińska et al. (2021) & HFD (60\%) pregnancy and lactation & Wistar rats-SD & $\begin{array}{l}\uparrow \text { Anhedonia and depressive-like behaviour in the FST and SPT } \\
\text { Transcriptomics changes in the prefrontal cortex }\end{array}$ \\
\hline de la Garza et al. (2019) & CAF, HFD and HSD & Wistar rats-SD & $\begin{array}{l}\uparrow \text { Anhedonia and depressive-like behaviour in the FST } \\
\uparrow \text { TBK1 after HSD in the hippocampus }\end{array}$ \\
\hline $\begin{array}{l}\text { Trujillo-Villarreal et al. } \\
\text { (2021) }\end{array}$ & CAF & Wistar rats-SD & $\begin{array}{l}\downarrow \text { Motivation for natural rewards on OCT, SPT, and SFT } \\
\downarrow \text { Frontomesocorticolimbic circuit volume } \\
\downarrow \text { Synaptic terminals in the hippocampus and nucleus accumbens } \\
\uparrow \text { GFAP in the hippocampus and hypothalamus } \\
\downarrow \text { Number of hippocampal cells } \\
\downarrow \text { Myelin in the dentate gyrus of hippocampus } \\
\uparrow \text { GluR1 and GluR2 subunits of AMPA receptors } \\
\downarrow \text { mGluR2 expression in the hippocampus }\end{array}$ \\
\hline \multicolumn{4}{|c|}{ Anxiety } \\
\hline $\begin{array}{l}\text { Bruce-Keller et al. } \\
\text { (2017) }\end{array}$ & HFD & $\begin{array}{l}\mathrm{C} 57 \mathrm{BL} / 6 \\
\text { mice-SD }\end{array}$ & $\begin{array}{l}\uparrow \text { Anxiety-like behaviour in the open field in males } \\
\uparrow \text { Marble buying in males; Changes in microbial diversity in males and females }\end{array}$ \\
\hline $\begin{array}{l}\text { Matuszewska et al. } \\
\text { (2021) }\end{array}$ & CAF & Wistar rats-SD & $\begin{array}{l}\text { At PND25, CAF offspring } \\
\uparrow \% \text { of fat content; sex-specific differences in glucose levels } \\
\uparrow \text { Serum IL-6, IL-10, and TNF- } \alpha \text { in females } \\
\text { Sex-specific differences in concentration of IL- } 6 \text { and TNF- } \alpha \text {, } \uparrow \text { in CAF females } \\
\uparrow \text { Serum IL-10 in males }\end{array}$ \\
\hline Sasaki et al. (2013) & Perinatal HFD & $\begin{array}{l}\text { Long Evans } \\
\text { rats-SD }\end{array}$ & $\begin{array}{l}\downarrow \text { Anxiety-like behaviour in adult animals } \\
\text { Selective alteration in the GR expression and inflammatory genes in the } \\
\text { hippocampus and amygdala } \\
\uparrow \text { Corticosterone after stressful challenges in females }\end{array}$ \\
\hline Sasaki et al. (2014) & Perinatal HFD & $\begin{array}{l}\text { Long Evans } \\
\text { rats-SD }\end{array}$ & $\begin{array}{l}\uparrow \text { Center entries (females) and } \uparrow \text { time spent in the center (males and females) in } \\
\text { the OF } \\
\uparrow \text { Open arm entries in the elevated plus maze (both sexes) } \\
\uparrow \text { GR transcript in the hippocampus of the females } \\
\downarrow \text { Hippocampal gene expression of NF- } \kappa \mathrm{B}, \mathrm{IL}-6, \mathrm{IkB} \alpha \text {, and MKP- } 1 \text { in females } \\
\downarrow \text { Gene expression of NF- } \kappa \mathrm{B} \text { and IL-1Ra in the amygdala of females }\end{array}$ \\
\hline Ribeiro et al. (2018) & CAF & Swiss mice-SD & $\begin{array}{l}\downarrow \text { Latency for the first transition in the light-dark test } \\
\uparrow \text { Number of transitions and time spent in the dark of light-dark test in males } \\
\downarrow \text { Play behaviour }\end{array}$ \\
\hline Wright et al. (2011) & $\begin{array}{l}\text { Pre-gestational, gestational and } \\
\text { lactational CAF diet }\end{array}$ & Wistar rats-SD & $\downarrow$ Anxiety in males shown by the OF test \\
\hline
\end{tabular}

AMPA, $\alpha$-amino-3-hydroxy-5-methyl-4-isoxazolepropionic acid; CAF, cafeteria diet; FST, forced swimming test; GFAP, glial fibrillary acidic protein; GluR, glutamate receptor; GR, glucocorticoid receptor; HFD, high-fat diet; HSD, high-sugar diet; IL, interleukin; IL-1Ra, interleukin-1 receptor antagonist; IkB $\alpha$, I-kappa-B-alpha; MKP-1, mitogen-activated protein kinase 1; NF-кB, nuclear factor kappa B; OCT, operant conditioning test; OF, open field; PND, postnatal day; SD, standard diet; SPT, sucrose preference test; SFT, suppressed feeding test; TKB1, TANK binding kinase-1; TNF- $\alpha$, tumor necrosis factor-alpha.

consumption and has translational potential (Matuszewska et al., 2021). Wright et al. showed that maternal consumption of a CAF impacts offspring behaviour, regardless of whether consumption occurs in the preconception period, during pregnancy, lactation, or all these periods (Wright et al., 2011). Interestingly, CAF during lactation caused a consistent anxiolytic effect in males as observed by the increase of entries into and more time spent on open arms in the elevated plus-maze and the reduced latency to enter the center in the open field test. It reinforces that the sex of the offspring is an important factor and should be taken into account when studying the effects of maternal obesity (Wright et al., 2011).

Otherwise, consuming CAF exclusively during pregnancy increased maternal behaviours such as licking pups, arched 
nursing, and nest building after 2- and 8-days following birth. Interestingly, even with increased maternal behaviour, the offspring demonstrated behavioural disturbances in adolescence such as increased anxiety-like manifestations during light-dark tests (Ribeiro et al., 2018).

Table 1 summarizes preclinical studies demonstrating maternal diet's impact on depressive and anxiety-like behaviours in the progeny.

\section{THE GUT-BRAIN AXIS DURING FETAL DEVELOPMENT}

Data presented so far reinforce a causative relationship between maternal diet and neurologic programming in offspring. However, the underlying mechanism involved in this phenomenon remains elusive. Despite that, the influence of maternal microbiota on the development of anxious behaviours in the offspring has emerged as a mechanism that is sufficient to disrupt behavioural function in murine offspring in a sex-specific manner (Bruce-Keller et al., 2017). It suggests that intestinal dysbiosis could link the effects of unhealthy modern diets to the increased prevalence of neurodevelopmental and childhood disorders.

In addition to fermenting non-digestible fibers from the diet, which increases energy extraction from food, the influence of the gut microbiota on the host's physiology has been gaining notoriety from studies that demonstrate a bi-directional relationship with the brain, a pathway coined as the gut-brain axis (Martin et al., 2018). Although this communication is not completely clear, three main pathways are proposed: neural signals carried out by the vagus nerve; chemical signals synthesized by the microbiota, as short-chain fatty acids (SCFA) or neurotransmitters; and immunomodulation exerted by microbiota and its products (Morais et al., 2021).

The main phyla of bacteria that colonize the human gut are Firmicutes and Bacteroidetes (Eckburg et al., 2005), but microbiota composition is extremely dynamic and altered even in physiological contexts such as pregnancy. For example, in the first trimester, the maternal gut microbiota is more similar to that of men and non-pregnant women, while Proteobacteria and Actinobacteria are expanded during the third trimester (Koren et al., 2012). Conversely, analysis from 1479 pregnant women found no differences compared to age-matched non-pregnant women, suggesting individual heterogeneity as the most relevant element for changes in gut microbiota during pregnancy (Yang et al., 2020). Even though, in animal studies, mice that received faecal transplantation from women in the third trimester, but not in the first, develop weight gain and insulin resistance (Koren et al., 2012). Also, an increase in Akkermansia, Clostridium, Bacteroides, and Bifidobacterium genera was found in C57BL/6 pregnant mice, supporting the idea of gut microbiome changes during pregnancy (Gohir et al., 2015). These changes during pregnancy do not influence only the mother, but also impact the offspring's neurodevelopment (Al Rubaye et al., 2021), e.g., altering the offspring's microglial gene signature: male offspring of germ-free dams showed changes in the expression of 1216 microglial genes near birth. In contrast, the major impact on female offspring was observed in adulthood with alterations in 433 genes expressed by microglia compared to the offspring of dams with intact gut microbiota (Thion et al., 2018). Therefore, the maternal gut microbiota can also influence the offspring's brain.

Changes in the microbiota are not limited to physiological situations such as pregnancy. In metabolic diseases such as obesity, there is evidence demonstrating changes in the bacterial profile, such as an increase in Firmicutes and a decrease in Bacteroidetes compared to their lean counterparts both in obese humans (Crovesy et al., 2020) and in HFD-fed animals (Jo et al., 2021). Indeed, the interaction between obesity and pregnancy causes specific changes that impact both mother and offspring's microbiota. Mice exposed to HFD before mating and during gestation had increased Akkermansia (phylum Firmicutes) and Bifidobacterium compared to lean pregnant animals (Gohir et al., 2015). Previous studies show that the type of birth delivery can profoundly impact the bacterial profile in children (Ferretti et al., 2018; García-Mantrana et al., 2020), even though bacterial colonization does not occur in the uterus (Perez-Muñoz et al., 2017). The cohort study carried out by Chu et al. (2016) showed differences in the intestinal microbiota of children of mothers who consumed HFD compared to balanced diets, which demonstrated that the maternal diet influences both the mother and the infant's bacterial profile.

Advances in the field have been showing how gut microbiota could influence the development of mood disorders such as anxiety and depression (Peirce and Alviña, 2019; Huang and $\mathrm{Wu}, 2021$ ). In a recent study, pregnant women had their microbiota sequenced through stool samples during the third trimester of pregnancy, and children's behaviour was analyzed at 2 years of age. Interestingly, increased alpha diversity, a measure of microbiota diversity, was related to less internalizing behaviour at 2 years of age, which can be associated with decreased anxiety behaviour. Furthermore, the butyrate-producing families Lachnospiraceae and Ruminococcaceae were found in mothers from children with normal behaviour. Prenatal exposure to healthy diets was also linked to the development of healthy behaviour in children (Dawson et al., 2021). However, few studies explore this mechanism in the context of maternal overnutrition and offspring's behaviour.

Animal models support that maternal consumption of unhealthy diets during pregnancy, such as HFD, is related to gut microbiota changes that can predispose behaviours related to anxiety and depression in the offspring (Buffington et al., 2016; Bruce-Keller et al., 2017). To isolate the effects of obesitymodified microbiota on offspring behaviour, gut microbiota was transplanted from HFD-fed animals to female mice with depleted microbiota before copulation. It resulted in altered offspring's microbiota and primed anxiety-like behaviour in males, but not in females. Male offspring from HFD transplanted dams also showed a pattern of depressive-like behaviour. Therefore, maternal microbiota influenced by the diet can lead to depressive and anxiety-like behaviours in the offspring in a sex-dependent manner (Bruce-Keller et al., 2017). 


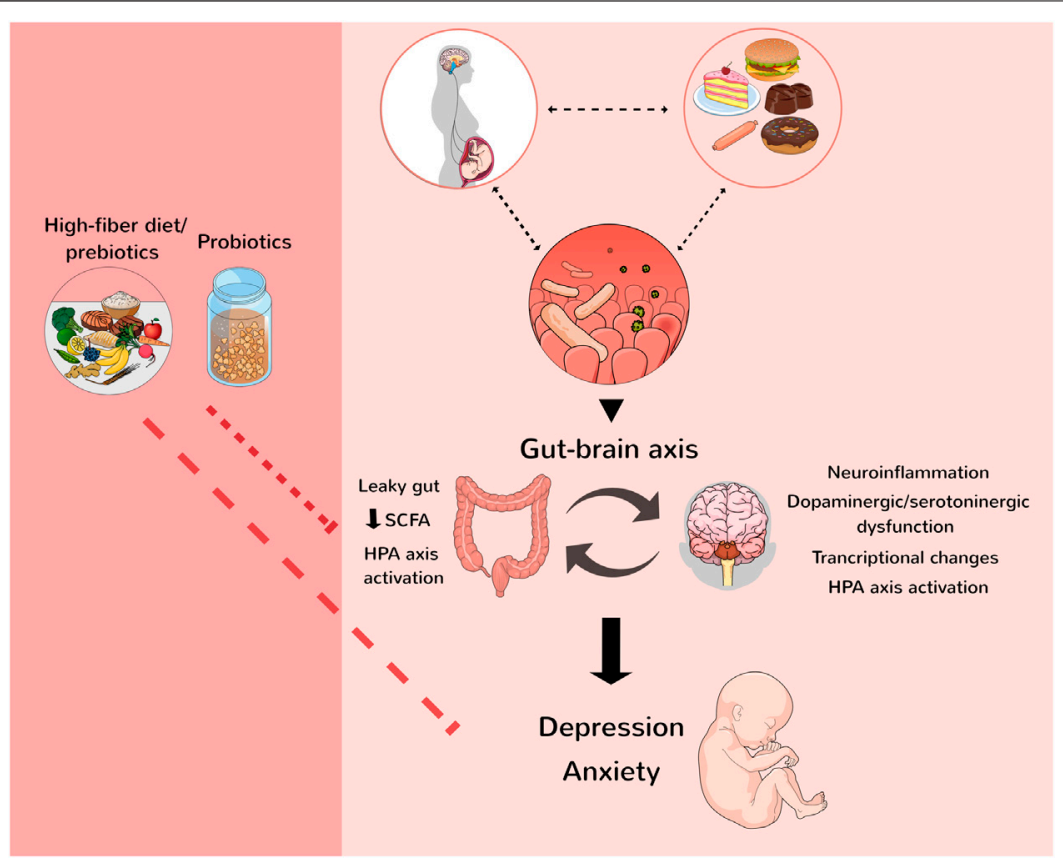

FIGURE 1 | Maternal diet can affect the offspring, increasing the chances of metabolic and neurodevelopmental diseases. The gut-brain axis modulates anxiety and depression development since maternal microbiota influence the offspring's behaviour. Some mechanisms showed in the figure are proposed to influence the development of neurological disorders such as psychiatric manifestations. The consumption of a high-fiber diet, prebiotics, and probiotics is helpful to modulate the offspring's microbiome and, thus, can diminish the risk of mental illnesses. HPA, hypothalamus-pituitary-adrenal; SCFA, short-chain fatty acids. Figure created by the authors with the assistance from Mind the Graph (mindthegraph.com).

In another preclinical study, co-housing the offspring of HFD dams with offspring of a control dam reversed changes in the offspring's microbiota caused by maternal obesogenic diet and alleviated the behavioural abnormalities. Moreover, faecal transplantation from offspring of HFD dams to germ-free mice led to behavioural effects similar to those observed in the offspring of obese dams (Buffington et al., 2016).

Among the proposed mechanisms, a decrease in fermenting bacteria responsible for SCFA production such as butyrate, propionate, and acetate can lead to a leaky gut, impairing the intestinal barrier and allowing immunogenic molecules from the gut, e.g., lipopolysaccharide (LPS) reach the circulation, where they activate inflammatory responses that can become chronic and lead to psychiatric manifestations (Dash et al., 2015; Winter et al., 2018). Yucatan pigs dams consuming CAF during pregnancy and lactation showed a decrease in SCFA in both dams' and offspring's faeces 100 days after birth. A decrease in neurogenesis markers in the offspring's hippocampus was also found, evidencing the involvement of the gut-brain axis in this context (Val-Laillet et al., 2017). Also, altered microbiota during gestation can trigger maternal immune activation, which leads to conditions in the offspring that also have anxiety as a feature, e.g., autism spectrum disorder (Careaga et al., 2017; Gottfried and Bambini-Junior, 2018). Important to mention, gut microbiota changes impact the synthesis of neurotransmitters such as serotonin, dopamine, and norepinephrine [for review see (Huang and $\mathrm{Wu}, 2021)$, which also might influence the development of mood disorders. Moreover, diet-induced maternal dysbiosis has also been proposed to increase hypothalamus-pituitary-adrenal (HPA) axis activation (Farzi et al., 2018; Di Gesù et al., 2021), whilst the activation of the HPA axis has an established relationship with psychiatric disorders such as anxiety (Frankiensztajn et al., 2020) (Figure 1).

Therefore, microbiota and its metabolites may provide an interesting mechanism of how the gut-brain axis is involved in the effects of obesity on the offspring's brain and also provide a potential therapeutic target. The use of prebiotics and probiotics has provoked interest in the scientific community in recent years. While probiotics can modulate the composition of intestinal bacteria, prebiotics are the substrate used for fermentation and production of metabolites with an interest to improve host health. Recent research data indicate positive impacts of these strategies regarding metabolic diseases and also in the CNS, including improvement of cognitive impairment, anxiety, and depression [for a review see (Liu et al., 2019; Paiva et al., 2020)]. The gestation period could be a suitable and opportune moment to introduce, or even prioritize the probiotics therapy to benefit the offspring. In an animal model, consumption of probiotics concomitant with an HFD started before pregnancy and continued until lactation was able to protect mice offspring against metabolic disturbances compared to offspring of dams on HFD alone (Guo et al., 2018). On the other hand, its effect in pregnant women is controversial since promising literature (Desai et al., 2021) contrasts with evidence such as a randomized clinical trial that found no 
benefits with Lactobacillus rhamnosus and Bifidobacterium lactis supplementation in obese pregnant women regarding depression and well-being (Dawe et al., 2020). Therefore, future studies are needed to robustly assert whether probiotic supplementation during pregnancy would be useful to prevent the development of mood disorders such as depression and anxiety in the progeny.

\section{CURRENT RESEARCH GAPS AND CONCLUSION}

As already mentioned, dysbiosis may be related to the genesis of chronic inflammation in obesity and, through the gut-brain axis, trigger neuroinflammation and the development of psychiatric manifestations (Peirce and Alviña, 2019; Milano et al., 2020; Shi et al., 2020). Maternal diet influences offspring's brain development, e.g., affecting microglial maturation and gene expression (Thion et al., 2018) and also gut microbiota composition (Buffington et al., 2016). Moreover, brain cells such as microglia can be influenced by the gut-brain axis acquiring a more pro-inflammatory profile and triggering behavioural disturbances (Duan et al., 2021).

In this review, we highlighted the influence of maternal overnutrition and obesity on offspring's CNS manifestations, mainly on depression and anxiety, psychiatric disorders increasingly prevalent in the world. We also show how the gut-brain axis is altered during pregnancy and obesity. Accordingly, healthier food consumption during pregnancy must be encouraged to prevent this global health issue (Hanson et al., 2017; Bhagavata Srinivasan et al., 2018).

Depression and anxiety may manifest at different stages of life, either in childhood, adulthood, or aging. Whenever it happens, the causes regarding maternal exposure cannot be excluded. Thus, a particularly productive focus for research would be to better understand the influence of the microbiota of obese mothers on the offspring's brain, allowing the establishment of

\section{REFERENCES}

Al Rubaye, H., Adamson, C. C., and Jadavji, N. M. (2021). The Role of Maternal Diet on Offspring Gut Microbiota Development: A Review. J. Neurosci. Res. 99, 284-293. doi:10.1002/jnr.24605

Aubuchon-Endsley, N., Morales, M., Giudice, C., Bublitz, M. H., Lester, B. M., Salisbury, A. L., et al. (2017). Maternal Pre-pregnancy Obesity and Gestational Weight Gain Influence Neonatal Neurobehaviour. Matern. Child Nutr. 13, e12317. doi:10.1111/mcn.12317

Barker, D., and Osmond, C. (1986). Infant Mortality, Childhood Nutrition, and Ischaemic Heart Disease in England and Wales. The Lancet 327, 1077-1081. doi:10.1016/s0140-6736(86)91340-1

Belujon, P., and Grace, A. A. (2017). Dopamine System Dysregulation in Major Depressive Disorders. Int. J. Neuropsychopharmacol. 20, 1036-1046. doi:10.1093/ijnp/pyx056

Bergmann, S., Schlesier-Michel, A., Wendt, V., Grube, M., Keitel-Korndörfer, A., Gausche, R., et al. (2016). Maternal Weight Predicts Children's Psychosocial Development via Parenting Stress and Emotional Availability. Front. Psychol. 7, 1156. doi:10.3389/fpsyg.2016.01156

Bhagavata Srinivasan, S. P., Raipuria, M., Bahari, H., Kaakoush, N. O., and Morris, M. J. (2018). Impacts of Diet and Exercise on Maternal Gut the importance of therapeutic interventions in this context. Modulating the microbiota through a healthier diet can be beneficial to the offspring, as maternal and offspring dietary fiber consumption was demonstrated to diminish cognitive and social disabilities while improving synaptic function and microglia maturation in the offspring (Liu et al., 2021). The use of prebiotics and probiotics has shown promising results to modulate the maternal and offspring microbiota, and it could be an interesting strategy to prevent psychiatric conditions, such as anxiety and depression (Sanders et al., 2019; Lee, 2021). However, conflicting results and the lack of knowledge about the mechanisms of action of these interventions should encourage future research focused on this subject.

\section{AUTHOR CONTRIBUTIONS}

Conceptualization, MG, VBJ, RPG, and LSF; writing and literature review, all the authors; critical revision of the manuscript, VB-J, RPG, and LSF; figure and table preparation, JJ and IDT. All authors have read, edited and approved the final version of the article.

\section{FUNDING}

Our research groups received financial support, over the years, from Conselho Nacional de Desenvolvimento Científico e Tecnológico (CNPq), Coordenação de Aperfeiçoamento de Pessoal de Nível Superior (CAPES), Fundação de Amparo à Pesquisa do Estado do Rio Grande do Sul (FAPERGS), Fundo de Incentivo à Pesquisa e Eventos do Hospital de Clínicas de Porto Alegre (FIPE/HCPA), Universidade Federal de Ciências da Saúde de Porto Alegre (UFCSPA), National Institute of Science and Technology on Neuroimmunomodulation (INCT-NIM) and Erasmus+ Funding Programme.
Microbiota Are Transferred to Offspring. Front. Endocrinol. 9, 716. doi:10.3389/fendo.2018.00716

Bruce-Keller, A. J., Fernandez-Kim, S.-O., Townsend, R. L., Kruger, C., Carmouche, R., Newman, S., et al. (2017). Maternal Obese-type Gut Microbiota Differentially Impact Cognition, Anxiety and Compulsive Behavior in Male and Female Offspring in Mice. PLoS One 12, e0175577. doi:10.1371/journal.pone.0175577

Buffington, S. A., Di Prisco, G. V., Auchtung, T. A., Ajami, N. J., Petrosino, J. F., and Costa-Mattioli, M. (2016). Microbial Reconstitution Reverses Maternal DietInduced Social and Synaptic Deficits in Offspring. Cell 165, 1762-1775. doi:10. 1016/j.cell.2016.06.001

Cárdenas-Tueme, M., Montalvo-Martínez, L., Maldonado-Ruiz, R., CamachoMorales, A., and Reséndez-Pérez, D. (2020). Neurodegenerative Susceptibility during Maternal Nutritional Programing: Are Central and Peripheral Innate Immune Training Relevant. Front. Neurosci. 14, 13. doi:10.3389/fnins.2020.00013

Careaga, M., Murai, T., and Bauman, M. D. (2017). Maternal Immune Activation and Autism Spectrum Disorder: From Rodents to Nonhuman and Human Primates. Biol. Psychiatry 81, 391-401. doi:10.1016/j.biopsych. 2016.10.020

Cattane, N., Räikkönen, K., Anniverno, R., Mencacci, C., Riva, M. A., Pariante, C. M., et al. (2021). Depression, Obesity and Their Comorbidity during Pregnancy: 
Effects on the Offspring's Mental and Physical Health. Mol. Psychiatry 26, 462-481. doi:10.1038/s41380-020-0813-6

Chen, C., Xu, X., and Yan, Y. (2018). Estimated Global Overweight and Obesity burden in Pregnant Women Based on Panel Data Model. PLoS One 13, e0202183. doi:10. 1371/journal.pone.0202183

Chu, D. M., Antony, K. M., Ma, J., Prince, A. L., Showalter, L., Moller, M., et al. (2016). The Early Infant Gut Microbiome Varies in Association with a Maternal High-Fat Diet. Genome Med. 8, 77. doi:10.1186/s13073-016-0330-z

Crovesy, L., Masterson, D., and Rosado, E. L. (2020). Profile of the Gut Microbiota of Adults with Obesity: a Systematic Review. Eur. J. Clin. Nutr. 74, 1251-1262. doi:10.1038/s41430-020-0607-6

Dash, S., Clarke, G., Berk, M., and Jacka, F. N. (2015). The Gut Microbiome and Diet in Psychiatry. Curr. Opin. Psychiatry 28, 1-6. doi:10.1097/YCO. 0000000000000117

Dawe, J. P., McCowan, L. M. E., Wilson, J., Okesene-Gafa, K. A. M., and Serlachius, A. S. (2020). Probiotics and Maternal Mental Health: A Randomised Controlled Trial Among Pregnant Women with Obesity. Sci. Rep. 10, 1291. doi:10.1038/ s41598-020-58129-w

Dawson, S. L., O’Hely, M., Jacka, F. N., Ponsonby, A.-L., Symeonides, C., Loughman, A., et al. (2021). Maternal Prenatal Gut Microbiota Composition Predicts Child Behaviour. EBioMedicine 68, 103400. doi:10.1016/j.ebiom.2021. 103400

de la Garza, A., Garza-Cuellar, M., Silva-Hernandez, I., Cardenas-Perez, R., ReyesCastro, L., Zambrano, E., et al. (2019). Maternal Flavonoids Intake Reverts Depression-like Behaviour in Rat Female Offspring. Nutrients 11, 572. doi:10. 3390/nu11030572

Desai, V., Kozyrskyj, A. L., Lau, S., Sanni, O., Dennett, L., Walter, J., et al. (2021). Effectiveness of Probiotic, Prebiotic, and Synbiotic Supplementation to Improve Perinatal Mental Health in Mothers: A Systematic Review and Meta-Analysis. Front. Psychiatry 12, 622181. doi:10.3389/fpsyt.2021.622181

Di Gesù, C. M., Matz, L. M., and Buffington, S. A. (2021). Diet-induced Dysbiosis of the Maternal Gut Microbiome in Early Life Programming of Neurodevelopmental Disorders. Neurosci. Res. 168, 3-19. doi:10.1016/j. neures.2021.05.003

Duan, C., Huang, L., Zhang, C., Zhang, L., Xia, X., Zhong, Z., et al. (2021). Gut Commensal-Derived Butyrate Reverses Obesity-Induced Social Deficits and Anxiety-like Behaviors via Regulation of Microglial Homeostasis. Eur. J. Pharmacol. 908, 174338. doi:10.1016/j.ejphar.2021.174338

Eckburg, P. B., Bik, E. M., Bernstein, C. N., Purdom, E., Dethlefsen, L., Sargent, M., et al. (2005). Diversity of the Human Intestinal Microbial flora. Science 308, 1635-1638. doi:10.1126/science.1110591

Edlow, A. G. (2017). Maternal Obesity and Neurodevelopmental and Psychiatric Disorders in Offspring. Prenat. Diagn. 37, 95-110. doi:10. 1002/pd.4932

Farzi, A., Fröhlich, E. E., and Holzer, P. (2018). Gut Microbiota and the Neuroendocrine System. Neurotherapeutics 15, 5-22. doi:10.1007/s13311017-0600-5

Ferretti, P., Pasolli, E., Tett, A., Asnicar, F., Gorfer, V., Fedi, S., et al. (2018). Mother-to-Infant Microbial Transmission from Different Body Sites Shapes the Developing Infant Gut Microbiome. Cell Host \& Microbe 24, 133-145. e5. doi:10.1016/j.chom.2018.06.005

Frankiensztajn, L. M., Elliott, E., and Koren, O. (2020). The Microbiota and the Hypothalamus-Pituitary-Adrenocortical (HPA) axis, Implications for Anxiety and Stress Disorders. Curr. Opin. Neurobiol. 62, 76-82. doi:10. 1016/j.conb.2019.12.003

Fusco, S., Spinelli, M., Cocco, S., Ripoli, C., Mastrodonato, A., Natale, F., et al. (2019). Maternal Insulin Resistance Multigenerationally Impairs Synaptic Plasticity and Memory via Gametic Mechanisms. Nat. Commun. 10, 4799. doi:10.1038/s41467-019-12793-3

García-Mantrana, I., Selma-Royo, M., González, S., Parra-Llorca, A., Martínez-Costa, C., and Collado, M. C. (2020). Distinct Maternal Microbiota Clusters Are Associated with Diet during Pregnancy: Impact on Neonatal Microbiota and Infant Growth during the First 18 Months of Life. Gut Microbes 11, 962-978. doi:10.1080/19490976.2020.1730294

Gawlinska, K., Gawlinski, D., Przegalinski, E., and Filip, M. (2019). Maternal High-Fat Diet during Pregnancy and Lactation Provokes Depressive-like Behavior and Influences the Irisin/brain-Derived Neurotrophic Factor axis and Inflammatory Factors in Male and Female Offspring in Rats. J. Physiol. Pharmacol. 70 (3), 407-417. doi:10.26402/jpp.2019.3.07

Gawlińska, K., Gawliński, D., Korostyński, M., Borczyk, M., Frankowska, M., Piechota, M., et al. (2021). Maternal Dietary Patterns Are Associated with Susceptibility to a Depressive-like Phenotype in Rat Offspring. Develop. Cogn. Neurosci. 47, 100879. doi:10.1016/j.dcn.2020.100879

Gohir, W., Whelan, F. J., Surette, M. G., Moore, C., Schertzer, J. D., and Sloboda, D. M. (2015). Pregnancy-related Changes in the Maternal Gut Microbiota Are Dependent upon the Mother's Periconceptional Diet. Gut Microbes 6, 310-320. doi:10.1080/19490976.2015.1086056

Gottfried, C., and Bambini-Junior, V. (2018). Insights into the Relationship of the Immune System with Neurodevelopmental and Psychiatric Disorders. Neuroimmunomodulation 25, 243-245. doi:10.1159/000496180

Guo, Y., Wang, Z., Chen, L., Tang, L., Wen, S., Liu, Y., et al. (2018). Diet Induced Maternal Obesity Affects Offspring Gut Microbiota and Persists into Young Adulthood. Food Funct. 9, 4317-4327. doi:10.1039/c8fo00444g

Hanson, M., Barker, M., Dodd, J. M., Kumanyika, S., Norris, S., Steegers, E., et al. (2017). Interventions to Prevent Maternal Obesity before conception, during Pregnancy, and post Partum. Lancet Diabetes Endocrinol. 5, 65-76. doi:10.1016/ S2213-8587(16)30108-5

Hatanaka, Y., Kabuta, T., and Wada, K. (2017). Disturbance in Maternal Environment Leads to Abnormal Synaptic Instability during Neuronal Circuitry Development. Front. Neurosci. 11, 35. doi:10.3389/fnins.2017.00035

Howell, K. R., and Powell, T. L. (2017). Effects of Maternal Obesity on Placental Function and Fetal Development. Reproduction 153, R97-R108. doi:10.1530/ REP-16-0495

Huang, F., and Wu, X. (2021). Brain Neurotransmitter Modulation by Gut Microbiota in Anxiety and Depression. Front. Cel Dev. Biol. 9, 649103. doi:10.3389/fcell.2021.649103

Jo, J.-K., Seo, S.-H., Park, S.-E., Kim, H.-W., Kim, E.-J., Kim, J.-S., et al. (2021). Gut Microbiome and Metabolome Profiles Associated with High-Fat Diet in Mice. Metabolites 11, 482. doi:10.3390/metabo1 1080482

Koren, O., Goodrich, J. K., Cullender, T. C., Spor, A., Laitinen, K., Kling Bäckhed, H., et al. (2012). Host Remodeling of the Gut Microbiome and Metabolic Changes during Pregnancy. Cell 150, 470-480. doi:10.1016/j.cell.2012.07.008

Kumpulainen, S. M., Girchenko, P., Lahti-Pulkkinen, M., Reynolds, R. M., Tuovinen, S., Pesonen, A.-K., et al. (2018). Maternal Early Pregnancy Obesity and Depressive Symptoms during and after Pregnancy. Psychol. Med. 48, 2353-2363. doi:10.1017/S0033291717003889

Lee, S.-K. (2021). Don't Worry, Heavy Moms; Just Eat Your Broccoli(or Kimchi)!. Mol. Cell 44, 422-424. doi:10.14348/molcells.2021.0118

Liu, R. T., Walsh, R. F. L., and Sheehan, A. E. (2019). Prebiotics and Probiotics for Depression and Anxiety: A Systematic Review and Meta-Analysis of Controlled Clinical Trials. Neurosci. Biobehavioral Rev. 102, 13-23. doi:10.1016/j. neubiorev.2019.03.023

Liu, X., Li, X., Xia, B., Jin, X., Zou, Q., Zeng, Z., et al. (2021). High-fiber Diet Mitigates Maternal Obesity-Induced Cognitive and Social Dysfunction in the Offspring via Gut-Brain axis. Cel Metab. 33, 923-938. e6. doi:10.1016/j.cmet. 2021.02.002

Martin, C. R., Osadchiy, V., Kalani, A., and Mayer, E. A. (2018). The Brain-GutMicrobiome Axis. Cell Mol. Gastroenterol. Hepatol. 6, 133-148. doi:10.1016/j. jcmgh.2018.04.003

Matuszewska, J., Zalewski, T., Klimaszyk, A., Ziarniak, K., Jurga, S., Chmurzynska, A., et al. (2021). Mothers' Cafeteria Diet Induced Sex-specific Changes in Fat Content, Metabolic Profiles, and Inflammation Outcomes in Rat Offspring. Sci. Rep. 11, 18573. doi:10.1038/s41598-021-97487-x

McDonald, S. D., McKinney, B., Foster, G., Taylor, V., Lutsiv, O., and Pullenayegum, E. (2015). The Combined Effects of Maternal Depression and Excess Weight on Neonatal Outcomes. Int. J. Obes. 39, 1033-1040. doi:10.1038/ijo.2015.44

Menting, M. D., van de Beek, C., Mintjens, S., Wever, K. E., Korosi, A., Ozanne, S. E., et al. (2019). The Link between Maternal Obesity and Offspring Neurobehavior: A Systematic Review of Animal Experiments. Neurosci. Biobehavioral Rev. 98, 107-121. doi:10.1016/j.neubiorev.2018.12.023

Milano, W., Ambrosio, P., Carizzone, F., De Biasio, V., Di Munzio, W., Foia, M. G., et al. (2020). Depression and Obesity: Analysis of Common Biomarkers. Diseases 8, 23. doi:10.3390/diseases8020023 
Morais, L. H., Schreiber, H. L., Mazmanian, S. K., and Mazmanian, S. K. (2021). The Gut Microbiota-Brain axis in Behaviour and Brain Disorders. Nat. Rev. Microbiol. 19, 241-255. doi:10.1038/s41579-020-00460-0

Naef, L., Moquin, L., Dal Bo, G., Giros, B., Gratton, A., and Walker, C.-D. (2011). Maternal High-Fat Intake Alters Presynaptic Regulation of Dopamine in the Nucleus Accumbens and Increases Motivation for Fat Rewards in the Offspring. Neuroscience 176, 225-236. doi:10.1016/j.neuroscience.2010.12.037

Naef, L., Srivastava, L., Gratton, A., Hendrickson, H., Owens, S. M., and Walker, C.D. (2008). Maternal High Fat Diet during the Perinatal Period Alters Mesocorticolimbic Dopamine in the Adult Rat Offspring: Reduction in the Behavioral Responses to Repeated Amphetamine Administration. Psychopharmacology 197, 83-94. doi:10.1007/s00213-007-1008-4

Ortiz-Valladares, M., Pedraza-Medina, R., Pinto-González, M. F., Muñiz, J. G., Gonzalez-Perez, O., and Moy-López, N. A. (2021). Neurobiological Approaches of High-Fat Diet Intake in Early Development and Their Impact on Mood Disorders in Adulthood: A Systematic Review. Neurosci. Biobehavioral Rev. 129, 218-230. doi:10.1016/j.neubiorev.2021.07.028

Paiva, I. H. R., Duarte-Silva, E., and Peixoto, C. A. (2020). The Role of Prebiotics in Cognition, Anxiety, and Depression. Eur. Neuropsychopharmacol. 34, 1-18. doi:10.1016/j.euroneuro.2020.03.006

Paradis, J., Boureau, P., Moyon, T., Nicklaus, S., Parnet, P., and Paillé, V. (2017). Perinatal Western Diet Consumption Leads to Profound Plasticity and GABAergic Phenotype Changes within Hypothalamus and Reward Pathway from Birth to Sexual Maturity in Rat. Front. Endocrinol. 8, 216. doi:10.3389/ fendo.2017.00216

Peirce, J. M., and Alviña, K. (2019). The Role of Inflammation and the Gut Microbiome in Depression and Anxiety. J. Neurosci. Res. 97, 1223-1241. doi:10. 1002/jnr.24476

Perez-Muñoz, M. E., Arrieta, M.-C., Ramer-Tait, A. E., and Walter, J. (2017). A Critical Assessment of the "Sterile Womb" and "In Utero Colonization" Hypotheses: Implications for Research on the pioneer Infant Microbiome. Microbiome 5, 48. doi:10.1186/s40168-017-0268-4

Rasmussen, J. M., Graham, A. M., Entringer, S., Gilmore, J. H., Styner, M., Fair, D. A., et al. (2019). Maternal Interleukin-6 Concentration during Pregnancy Is Associated with Variation in Frontolimbic white Matter and Cognitive Development in Early Life. Neuroimage 185, 825-835. doi:10.1016/j. neuroimage.2018.04.020

Ribeiro, A. C. A. F., Batista, T. H., Veronesi, V. B., Giusti-Paiva, A., and Vilela, F. C. (2018). Cafeteria Diet during the Gestation Period Programs Developmental and Behavioral Courses in the Offspring. Int. J. Dev. Neurosci. 68, 45-52. doi:10. 1016/j.ijdevneu.2018.05.001

Rivera, H. M., Christiansen, K. J., and Sullivan, E. L. (2015). The Role of Maternal Obesity in the Risk of Neuropsychiatric Disorders. Front. Neurosci. 9, 194. doi:10.3389/fnins.2015.00194

Sanders, A., Rackers, H., and Kimmel, M. (2019). A Role for the Microbiome in Mother-Infant Interaction and Perinatal Depression. Int. Rev. Psychiatry 31, 280-294. doi:10.1080/09540261.2018.1548431

Sasaki, A., de Vega, W. C., St-Cyr, S., Pan, P., and McGowan, P. O. (2013). Perinatal High Fat Diet Alters Glucocorticoid Signaling and Anxiety Behavior in Adulthood. Neuroscience 240, 1-12. doi:10.1016/j.neuroscience.2013.02.044

Sasaki, A., de Vega, W., Sivanathan, S., St-Cyr, S., and McGowan, P. O. (2014). Maternal High-Fat Diet Alters Anxiety Behavior and Glucocorticoid Signaling in Adolescent Offspring. Neuroscience 272, 92-101. doi:10.1016/j.neuroscience. 2014.04.012

Schmitz, L., Kuglin, R., Bae-Gartz, I., Janoschek, R., Appel, S., Mesaros, A., et al. (2018). Hippocampal Insulin Resistance Links Maternal Obesity with Impaired Neuronal Plasticity in Adult Offspring. Psychoneuroendocrinology 89, 46-52. doi:10.1016/j.psyneuen.2017.12.023

Shi, H., Wang, Q., Zheng, M., Hao, S., Lum, J. S., Chen, X., et al. (2020). Supplement of Microbiota-Accessible Carbohydrates Prevents Neuroinflammation and Cognitive Decline by Improving the Gut Microbiota-Brain axis in DietInduced Obese Mice. J. Neuroinflammation 17, 77. doi:10.1186/s12974-02001760-1
Smith, B. L., and Reyes, T. M. (2017). Offspring Neuroimmune Consequences of Maternal Malnutrition: Potential Mechanism for Behavioral Impairments that Underlie Metabolic and Neurodevelopmental Disorders. Front. Neuroendocrinology 47, 109-122. doi:10.1016/j.yfrne. 2017.07.007

Sullivan, E. L., Riper, K. M., Lockard, R., and Valleau, J. C. (2015). Maternal HighFat Diet Programming of the Neuroendocrine System and Behavior. Horm. Behav. 76, 153-161. doi:10.1016/j.yhbeh.2015.04.008

Thion, M. S., Low, D., Silvin, A., Chen, J., Grisel, P., Schulte-Schrepping, J., et al. (2018). Microbiome Influences Prenatal and Adult Microglia in a Sex-specific Manner. Cell 172, 500-516. e16. doi:10.1016/j.cell.2017.11.042

Tong, L., and Kalish, B. T. (2021). The Impact of Maternal Obesity on Childhood Neurodevelopment. J. Perinatol. 41, 928-939. doi:10.1038/s41372-020-00871-0

Trujillo-Villarreal, L. A., Romero-Díaz, V. J., Marino-Martínez, I. A., Fuentes-Mera, L., Ponce-Camacho, M. A., Devenyi, G. A., et al. (2021). Maternal Cafeteria Diet Exposure Primes Depression-like Behavior in the Offspring Evoking Lower Brain Volume Related to Changes in Synaptic Terminals and Gliosis. Transl. Psychiatry 11, 53. doi:10.1038/s41398-02001157-x

Tye, K. M., Mirzabekov, J. J., Warden, M. R., Ferenczi, E. A., Tsai, H.-C., Finkelstein, J., et al. (2013). Dopamine Neurons Modulate Neural Encoding and Expression of Depression-Related Behaviour. Nature 493, 537-541. doi:10. 1038/nature 11740

Val-Laillet, D., Besson, M., Guérin, S., Coquery, N., Randuineau, G., Kanzari, A., et al. (2017). A Maternal Western Diet during Gestation and Lactation Modifies Offspring's Microbiota Activity, Blood Lipid Levels, Cognitive Responses, and Hippocampal Neurogenesis in Yucatan Pigs. FASEB j. 31, 2037-2049. doi:10. 1096/fj.201601015R

WHO (2017). Depression and Other Common Mental Disorders: Global Health Estimates. World Health Organization. Available at: https://apps.who.int/iris/ bitstream/handle/10665/254610/W? sequence=1 (Accessed October 7, 2021).

Winter, C., von Rumohr, A., Mundt, A., Petrus, D., Klein, J., Lee, T., et al. (2007). Lesions of Dopaminergic Neurons in the Substantia Nigra Pars Compacta and in the Ventral Tegmental Area Enhance Depressive-like Behavior in Rats. Behav. Brain Res. 184, 133-141. doi:10.1016/j.bbr.2007.07.002

Winter, G., Hart, R. A., Charlesworth, R. P. G., and Sharpley, C. F. (2018). Gut Microbiome and Depression: what We Know and what We Need to Know. Rev. Neurosci. 29, 629-643. doi:10.1515/revneuro-2017-0072

Wright, T., Langley-Evans, S. C., and Voigt, J.-P. (2011). The Impact of Maternal Cafeteria Diet on Anxiety-Related Behaviour and Exploration in the Offspring. Physiol. Behav. 103, 164-172. doi:10.1016/j.physbeh.2011.01.008

Yang, H., Guo, R., Li, S., Liang, F., Tian, C., Zhao, X., et al. (2020). Systematic Analysis of Gut Microbiota in Pregnant Women and its Correlations with Individual Heterogeneity. NPJ Biofilms Microbiomes 6, 32. doi:10.1038/s41522020-00142-y

Conflict of Interest: The authors declare that the research was conducted in the absence of any commercial or financial relationships that could be construed as a potential conflict of interest.

Publisher's Note: All claims expressed in this article are solely those of the authors and do not necessarily represent those of their affiliated organizations, or those of the publisher, the editors and the reviewers. Any product that may be evaluated in this article, or claim that may be made by its manufacturer, is not guaranteed or endorsed by the publisher.

Copyright (c) 2022 Jantsch, Tassinari, Giovenardi, Bambini-Junior, Guedes and de Fraga. This is an open-access article distributed under the terms of the Creative Commons Attribution License (CC BY). The use, distribution or reproduction in other forums is permitted, provided the original author(s) and the copyright owner(s) are credited and that the original publication in this journal is cited, in accordance with accepted academic practice. No use, distribution or reproduction is permitted which does not comply with these terms. 